\section{Cutaneous Leishmaniasis, Northern Afghanistan}

To the Editor: In Afghanistan, most cutaneous leishmaniasis cases are caused by Leishmania tropica, which is transmitted anthroponotically by the sandfly Phlebotomus sergenti (1). Cutaneous leishmaniasis can have devastating effects on local communities because of its clinical symptoms, i.e., large, multiple, or both, disfiguring lesions, that can lead to social ostracism of affected persons (e.g., women are often deemed unsuitable for marriage or to raise children) (2). Cutaneous leishmaniasis is considered a low priority disease by international donor agencies because treatment costs are high and the disease does not cause death (3).

Data on the effects of cutaneous leishmaniasis in Afghanistan previously have been available only for Kabul ciry; recent studies have reported an estimated 67,500 cases (4). Because of the migration of an estimated 4.5 million infected Afghan refugees returning home from other countries, the sporadic treatment of patients infected with cutaneous leishmaniasis, and limited control of the sandfly vector, $L$. tropica has spread to areas that were previously nonendemic for the disease, e.g., northeastern Afghanistan.

A survey in Faizabad city, Badakhshan Province, was conducted in June 2003 by HealthNet International to collect data on the impact of cutaneous leishmaniasis. Leishmaniasis in this region is transmitted from April to October. The city was divided into 10 districts, and 20 households were surveyed along a randomly chosen transect drawn from the center of each district. A team of experienced medical staff clinically diagnosed cutaneous leishmaniasis (based on the presence or absence of cutaneous leishmaniasis lesions or scars, number of lesions, date of lesion onset) in household members and interviewed them to collect demogyraphic data (gender, age). Becau se of logistic constraints, parasitolog ic diagnosis of cutaneous leishmania is lesions (i.e., microscopic examination or parasite culture) was not conducted. However, in Afgha istan, skin lesions attributed to causes other than cutancous leishmaniasis are rare, and experience has shown that clinical diagnosis has a sensitivity and specificity of $>80 \%$ and $>0 \%$, respectively (Reithinger et al., unpub. data). Written approval to conduct the study was obtained fiom the Ministry of Health. Informed consent was obtained from study participants; all study participants with active cases of the disease were offered free anti-leishmanial treatment at the HealthNet International leishmaniasis clinic

We surveyed 1,832 people from 200 houscholds; 8.3\% (152/1,832) and $7.8 \%(142 / 1,832)$ had active cutaneous leishmaniasis lesions or scars, respecively. Of those persons with cutane ous leishmaniasis lesions, the mean lesion number was 2.4 (range 1-14), the mean lesion size was 2.4 $\mathrm{cm}$ (ra uge 1-5.5), and the mean lesion duration (to survey date) was 5.6 month: (range 1-11). Active prevalence ivas not associated with gender (Yates corrected $\chi^{2}=2.16, p=0.14$ ); $85 / 152(56 \%)$ of the cutancous leishmanias is case-patients were women, and $67 / 152$ (44\%) of culaneous leishmanias:is case-patients were men. Data showed that persons aged $\leq 15$ years vere at higher risk of contracting the disease than were persons aged $=15$ years (odds ratio $=2.23$, $95 \%$ C I 1.54 to 3.24 , Yates-corrected $\left.\chi^{2}=1^{(1} .44, p<0.001\right)$

Based on population estimates of 65,000 people and observed prevalence, ipproximately 5,395 culaneous leishm ıniasis case-patients would be found in Faizabad. The low preva- lence of scars, compared to the high prevalence of disease, shows that cutaneous leishmaniasis has been introduced into Faizabad only recently $(1,4)$. Local Ministry of Health records show that the disease was virtually absent ( $<50$ annual cases) in Badakhshan 3 years ago; this information is corroborated by the observation that the mean time since the recovery of surveyed people with cutaneous leishmaniasis scars was 1.5 years (range 0.3-15). Although no attempts were made to identify circulating Leishmania sp., the current epidemic is likely caused by $L$. tropica because both men and women are equally affected and younger age gtoups are at higher risk for cutaneous leishmaniasis than older age groups $(1,4)$. Current analyses are under way to establish risk factors (e.g., presence or absence of animals, type of house construction) for contracting the diseàse.

With support from HealthNet International, three leishmaniasis clinics have been established in Faizabad to increase the total number of patients whose illness is diagnosed and treated; to reduce the risk to susceptible persons through the subsidized sale of insecticide-impregnated bed nets; to train and supervise the Ministry of Health staff in diagnosis, treatment, and prevention of the disease; and to implement health education campaigns for patients attending the clinics and the community at large. Hopefully, these activities will prevent the current cutaneous leishmaniasis outbreak from becoming an epidemic, as it has been in Kabul over the past 15 years $(4,5)$.

\section{Acknowledgments}

We thank the $\Lambda$ fghan Ministry of Health and the HealthNet International survey team staff for logistical support.

This study was supported by the United Nations Mission to $\Lambda$ fghanistan.

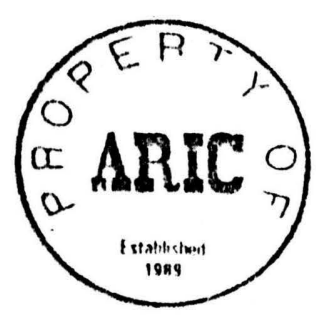

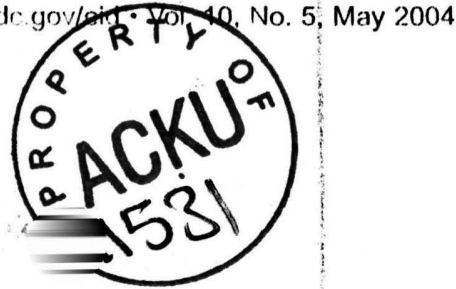




\section{Richard Reithinger, ${ }^{*} \dagger$ Khoksar Aadil, $\uparrow$ Samad Hami, $\uparrow$ and Jan Kolaczinski $\boldsymbol{\dagger}$}

*London School of Hygiene and Tropical Medicine, London, United Kingdom; and †HealthNet International, Peshawar, Pakistan

\section{References}

1. Ashford R, Kohestany K, Karimzad M. Cutaneous leishmaniasis in Kabul: observations on a prolonged epidemic. Ann Trop Med Parasitol 1992;86:361-71.

2. Griffiths WDA. Old World cutaneous leishmaniasis. In: Peters W, Killick-Kendrick R, editors. The leishmaniases in biology and medicine. London: Academic Press; 1987. p. 617-43.

3. Trouiller P, Torreele E, Olliaro P, White N, Foster S, Wirth D, et al. Drugs for neglected diseases: a failure of the market and a public health failure? Trop Med Int Health 2001;6:945-51.

4. Reithinger R, Mohsen M, Aadil K, Sidiqi M, Erasmus P, Coleman PG. The burden of anthroponotic cutaneous leishmaniasis in Kabul, Afghanistan. Emerg Infect Dis 2003;9:727.

5. Reyburn H, Rowland M, Mohsen M, Khan $\mathrm{B}$, Davies CR. The prolonged epidemic of anthroponotic cutaneous lẹishmaniasis in Kabul, Afghanistan: "bringing down the neighbourhood." Trans R Soc Trop Med Hyg 2003;97,170-6.

Address for correspondence: Richard Reithinger, Disease Control and Vector Biology Unit, Department of Infectious and Tropical Medicine, London School of Hygiene and Tropical Medicine, Keppel Street, London WC1E 7HT, UK; fax: +44 207927 2164; email: rreithinger@yahoo.co.uk

\section{Rickettsia felis, Bartonella henselae, and B. clarridgeiae, New Zealand}

To the Editor: The cat flea (Ctenocephalides felis felis Bouché, 1935) is a ubiquitous parasite of domestic and wild animals that also feeds readily on people. Recent studies have implicated the cat flea as a vector of new and emerging infectious diseases (1). To determine the pathogens in C. felis in New Zealand, we collected 3 cat fleas from each of 11 dogs and 21 cats at the Massey University Veterinary Teaching Hospital from May to June 2003. The fleas were stored in $95 \%$ alcohol until they were identified by using morphologic criteria and washed in sterile phosphate-buffered saline. The DNA from each flea was extracted individually by using the QiaAmp Tissue Kit (QIAGEN Ltd., Hilden, Germany), according to the manufacturer's instructions. When polymerase chain reaction (PCR) was performed with primers for glt $\mathrm{A}$ and $r O m p \mathrm{~A}$ as described (2), products were obtained with DNA from $15(15 \%)$ of the fleas. The sequences of the products were identical to those of Rickettsia felis (GenBank AF191026) with infected fleas taken from both dogs $(3 / 11$; $27 \%)$ and cats $(7 / 21 ; 33 \%)$. When PCR was performed with primers for the 16S-23S rDNA interspacer region as described (3), products were obtained with DNA of four fleas. The sequences of the products from three fleas (from two cats) were identical to that of Bartonella henselae (GenBank AF312495), and the sequence of the product of one flea (from a cat) was identical to that of $B$. clarridgeiae (GenBank AF167989).

Our study is the first to identify $R$. felis in Oceania. The organism is a recently described human pathogen, and infections with this spotted fever group rickettsia have already been reported in 11 persons: 4 persons in the United States, 2 persons in Brazil, 4 persons in Europe, and 1 person in Thailand. The symptoms of the patients were nonspecific and included fever, headache, and rash. Diagnoses were made by sequencing products obtained by PCR with primers for the $17-\mathrm{kDa}$ protein (4), citrate synthase (4), and PS 120 protein (5) genes. $R$. felis has been established in tissue culture (XTC-2 and Vero cells) (6), and serologic testing has been used to diagnose infections (5). Reports indicate that patients respond rapidly to doxycycline therapy (5), and in vitro studies have shown the organism is susceptible to rifampin, thiamphenicol, and fluoroquinolones.

$B$. henselae is an agent of catscratch disease, bacillary angiomatosis, bacillary peliosis, endocarditis, bacteremia, and various neurologic and ocular conditions. Cats are the reservoir hosts, and contact with cats and their fleas is an established risk factor for most infections. Although $B$. henselae has been isolated from $17 \%$ of domestic cats in New Zealand (7), only two human infections have been reported in the country; neuroretinitis was diagnosed in both patients (8). In neighboring Australia, however, cat-scratch disease, bacillary angiomatosis, and endocarditis have been diagnosed in numerous patients. Cats are also the reservoir hosts of $B$. clarridgeiae which has been implicated as an agent of cat-scratch disease in humans and aortic valve endocarditis and hepatic disease in dogs (9). The organism has been found in cat fleas (as great as 17\%) in Europe (1), and although we found only one flea infected with $B$. clarridgeiae in New Zealand, this description is the first of the organism in Oceania. However, $B$. clarridgeiae has been found in domestic cats in nearby Indonesia and the Philippines (10).

Our findings add to the accumulating data on $R$. felis, B. henselae, and $B$. clarridgeiae and should alert medical workers in New Zealand, a common tourist destination, to the possibility that their patients may be infect-

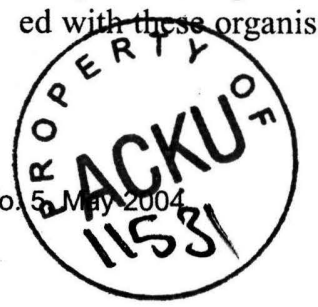

